\title{
Guidelines and Best Practice Recommendations on Obstetric Care of Pregnant Patients with COVID-19 Infection: Scoping Review
}

\author{
Wondimu Gudu Jeldu, MD, MPH*, Lemi Belay Tolu, MD and Abdulfetah Abdulkadir, MD \\ Department of Obstetrics and Gynecology, Saint Paul's Hospital Millennium Medical College, \\ Addis Ababa, Ethiopia
}

\begin{abstract}
Background: The Management of pregnant women with COVID-19 infection is usually based on limited evidence from care reports and expert opinions. The aim of this scoping review is to identify available guidelines and practice recommendations on obstetric care of pregnant women with COVID-19.

Methods: We searched guideline databases and websites of professional associations and international organizations working on sexual and reproductive health; We looked for guidelines, protocols, consensus statements and practice recommendations on management of pregnant women with COVID-19. Additionally, we searched: MEDLINE, EMBASE and Google Scholar. Data extraction was done by two independent reviewers using a customized tool that was developed to record the key information relevant to the review question. The difference between the two authors on data extraction was resolved by discussion.

Results: The antenatal care for COVID-19 positive mothers should be delayed until they test negative twice. Anatomic scanning may be considered in women who had the infection in the first trimester. Growth monitoring is recommended every 2-4 wks. Antenatal corticosteroids can be administered in those women with preterm pregnancy $(<34$ wks $)$ and mild illness. Low dose Aspirin can be continued safely. Tocolytics, particularly NSAIDs should be used cautiously. COVID-19 infection is not an indication for delivery. Termination is recommended in those with organ failure and in pts with severe respiratory failure. A single, asymptomatic or screen negative birth partner be permitted to stay with the woman. Continuous electronic fetal monitoring in in labor is recommended. Cesarean delivery only in those with severe respiratory distress on ventilators and those with organ failure. Shortening of the second stage is considered in COVID-19 parturients who are on face masks. There are no consistent recommendations on immediate cord clamping and options of breast feeding. Universal isolation of mother from the neonate is not a standard of practice. Postpartum care can be provided with telemedicine.
\end{abstract}

Conclusions: Obstetric care provision for COVID-19 infected mothers is primarily based on limited experience from case reports and expert opinions. Updated guidance for clinical practice are imperative as new scientific evidence emerges.

Keywords

COVID-19, Pregnancy, Labor, Delivery, Postpartum, Care, Review

\begin{abstract}
Abbreviations
ANC: Antenatal Care; ACOG: American College of Obstetrics and Gynecology; COVID-19: Corona Virus Disease-19; CSOG: Chinese Society of Obstetrics \& Gynecology; FIGO: Federation International Obstetrics \& Gynecology; ISUOG: International Society of Ultrasound in Obstetrics and Gynecology; ICM: International Confederation of Midwives; SOGC: Canadian Society of Obstetrics and Gynecology; SMFM: Society of Maternal Fetal Medicine; CDC: Center for Disease Control; RCOG: Royal College of Obstetrics and Gynecology; WHO: World Health Organization
\end{abstract}

\section{Background}

The 2019-20 coronavirus pandemic is an ongoing pandemic of coronavirus disease 2019 (COVID-19), caused by severe acute respiratory syndrome coronavirus 2 (SARS-CoV-2) [1]. The World Health Organization (WHO) declared the outbreak to be a Public Health Emergency of International Concern on
30 January 2020 and recognized it as a pandemic on 11 March $2020[2,3]$. The WHO considered reproductive health services, including care during pregnancy and child breath as essential health service to continue during the COVID-19 pandemics [4].

Changes in pregnant women's bodies and immune systems might expose pregnant women and their newborn to be

Copyright: (C) 2021 Jeldu WG, et al. This is an open-access article distributed under the terms of the Creative Commons Attribution License, which permits unrestricted use, distribution, and reproduction in any medium, provided the original author and source are credited. 


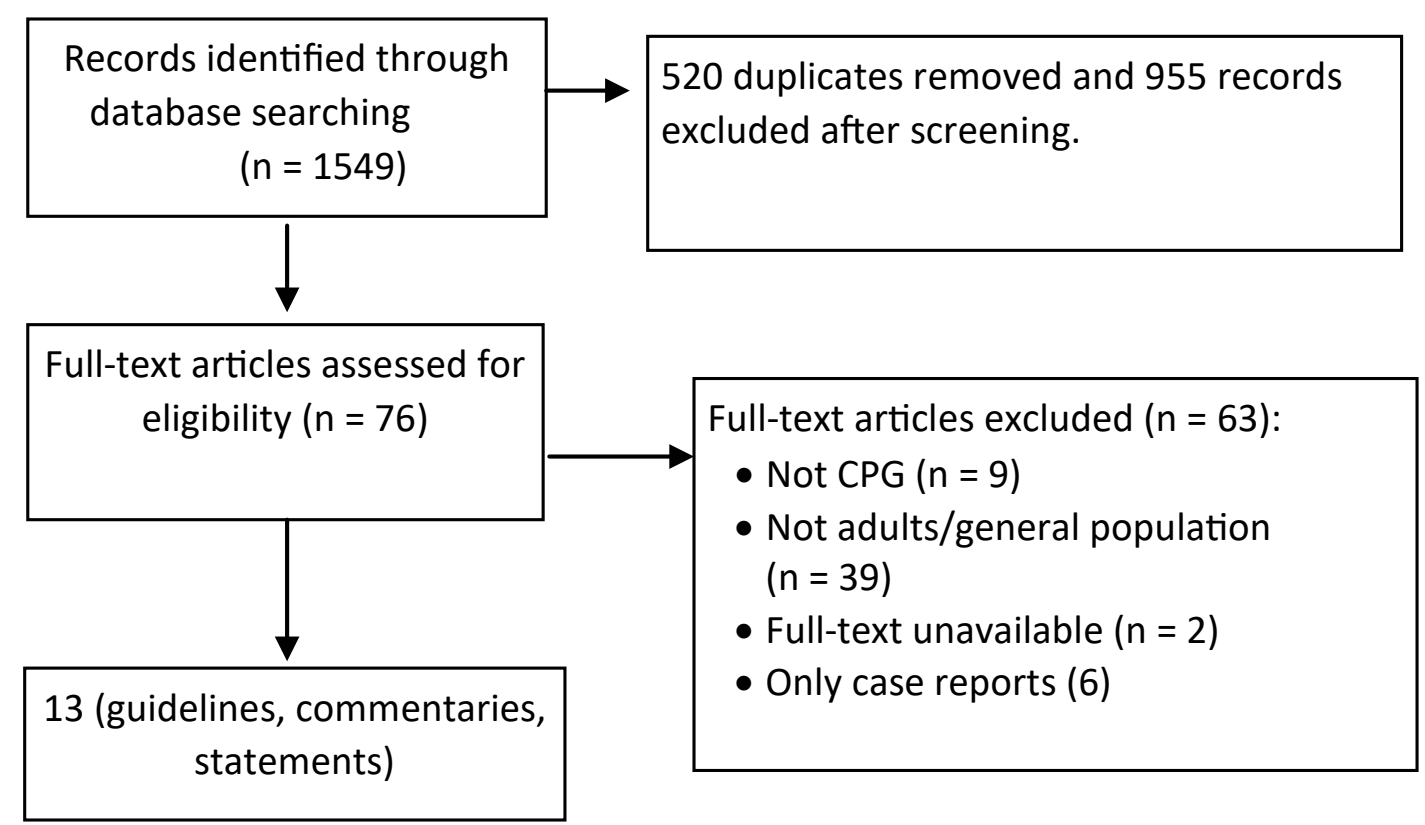

Figure 1: PRISMA flow diagram for selection process of resources.

badly affected by some respiratory infections like SARS-CoV-2. Different individual case reports, case series and studies with limited number of participants showed that there is no difference between the clinical manifestations of COVID-19 pregnant and non-pregnant women or adults of reproductive age [5-7]. There are different recommendations and consensus statements regarding the obstetric management of pregnant women with COVID-19 infection which are mainly based on limited case reports, case series and expert opinions.

The aim of this scoping review is therefore to identify available guidelines and practice recommendations on the Obstetric management of pregnant women with COVID-19. Since not many studies have been published addressing management of SARS-CoV-2 in pregnant women, the findings of this study will contribute to the scientific literature by providing valuable information. The Review questions were:

- What are the recommended approaches on antenatal care provision to pregnant women with COVID-19?

- What is the best practice in management of labor and delivery in women with COVID-19?

- What are practice recommendations regarding postpartum care, maternal isolation and breast feeding of neonates in women with COVID-19?

\section{Methods}

We searched for international professional associations, governmental and non-governmental organizations guidelines, protocols and practice recommendations on obstetric management of pregnant women with COVID-19 infection. We looked for guideline databases and their websites. We searched: World Health Organization (WHO), America College of Obstetrics and Gynecology (ACOG), Royal College of Obstetrics and Gynecology (RCOG), Royal College of Midwives (RCM), International Society of Ultrasound in Obstetrics and
Gynecology (ISUOG), International Federation of Obstetrics and Gynecology (FIGO), Society of Maternal and Fetal Medicine (SMFM), Society of Obstetrics and Gynecology of Canada (SOGC), International Confederation of Midwives (ICM), Chinese Society of Obstetrics \& Gynecology (CSOG).

In addition to searching the above professional associations and organizations guidelines, data bases and websites, we also developed search strategy (Appendix 1: Figure 1 Search strategy) to look any relevant emerging practice recommendations not endorsed by associations and organizations. We searched the following data bases: MEDLINE, EMBASE, Google Scholar and Cochrane Central Register of Controlled Trials (CENTRAL).

We included records labelled guideline, or recommendation, or consensus, or practice parameter, or position papers on obstetric (maternal) care of pregnant women with COVID-19 infection. While looking for our information sources we included all guidelines, defined as documents that aimed to state recommendations, that fulfilled the following criteria: Assessed antenatal care, labor and delivery, postnatal care, maternal separation; were published in the last 1 year (December 2019 to April 2020); full-text were available

*Corresponding author: Wondimu Gudu Jeldu, MD, MPH, Department of Obstetrics and Gynecology, Saint Paul's Hospital Millennium Medical College, Swaziland Street, Addis Ababa, 1271, Ethiopia, Tel: +251-911-406-475

Accepted: March 18, 2021

Published online: March 20, 2021

Citation: Jeldu WG, Tolu LB, Abdulkadir A (2021) Guidelines and Best Practice Recommendations on Obstetric Care of Pregnant Patients with COVID-19 Infection: Scoping Review. Annals Gynecol Obstet 5(1):110-117 
Citation: Jeldu WG, Tolu LB, Abdulkadir A (2021) Guidelines and Best Practice Recommendations on Obstetric Care of Pregnant Patients with COVID-19 Infection: Scoping Review. Annals Gynecol Obstet 5(1):110-117

in English; and used some form of evidences to guide their recommendations.

Data was extracted using a customized tool that was developed to record the key information of the source that's relevant to the review question (Appendix 2: Data Extraction form). Data were extracted by two independent authors. The following characteristics were extracted from the documents: authors; year of publication, affiliations, areas of obstetric service provision addressed and recommendations (Appendix 3 ). Differences between authors were resolved by discussion without third party involvement.

\section{Results}

We review 20 websites and the data base search yielded a total of 1549 records. After screening the titles and abstracts, 74 papers were retained for full-text review. Based on pre-defined inclusion criteria, 17 guidelines were included in the scoping review (Figure 1).

The characteristics of the guidelines, consensus statements and commentaries are summarized in Table 1 . Most were documents issued by different professional societies primarily Obstetrics \& Gynecology, one is consensus state-

Table 1: Characteristics of the documents and common practice recommendation areas.

\begin{tabular}{|c|c|c|c|c|}
\hline \multicolumn{2}{|c|}{ Practice area and common recommendations } & $\begin{array}{l}\text { Number of } \\
\text { records }\end{array}$ & $\begin{array}{l}\text { Sources and name of the } \\
\text { record }\end{array}$ & $\begin{array}{l}\text { Types of the } \\
\text { document (guideline, } \\
\text { commentaires, position } \\
\text { statements) }\end{array}$ \\
\hline \multirow{9}{*}{ 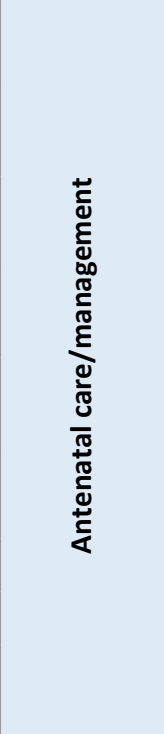 } & $\begin{array}{l}\text { Antenatal care to those who test } \\
\text { positive }\end{array}$ & 2 & SOGC, WHO & $\begin{array}{l}\text { Guidelines, position } \\
\text { statement }\end{array}$ \\
\hline & $\begin{array}{l}\text { Visits in those who recover from Covid } \\
\text { infection }\end{array}$ & 3 & $\begin{array}{l}\text { FIGO, WHO, China } \\
\text { Gyneology society consensus }\end{array}$ & $\begin{array}{l}\text { Guidelines, position } \\
\text { statements }\end{array}$ \\
\hline & Antenatal surveillance & 6 & $\begin{array}{l}\text { RCOG, FIGO, WHO, SOGC, } \\
\text { ISUOG }\end{array}$ & Guidelines, Statements \\
\hline & Anatomic scanning & 2 & FIGO, China Consensus & $\begin{array}{l}\text { Guidance, consensus } \\
\text { statemenet }\end{array}$ \\
\hline & Growth Monitoring & 6 & $\begin{array}{l}\text { RCOG, ICM, SOGC, FIGO, } \\
\text { china consensus, ISUOG }\end{array}$ & $\begin{array}{l}\text { Guidance, position } \\
\text { statements }\end{array}$ \\
\hline & Antenatal Corticosteroids & 4 & FIGO, ISUOG, SMFM, & $\begin{array}{l}\text { Guidance, position } \\
\text { statements }\end{array}$ \\
\hline & Use of NSAIDs (Asprin) & 1 & SMFM & Guidance \\
\hline & Use of Tocolytics & 2 & ISUOG, SMFM & Guidance \\
\hline & $\begin{array}{l}\text { Attendant with pregnant mother } \\
\text { (limit/screen status) }\end{array}$ & 3 & RCOG, FIGO, MFM guidance & Guidance \\
\hline \multirow{8}{*}{ 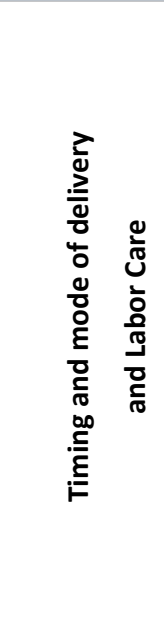 } & $\begin{array}{l}\text { Timing of delivery (in both mild and } \\
\text { severely ill) }\end{array}$ & 4 & $\begin{array}{l}\text { WHO, China consensus, ICM, } \\
\text { FIGO }\end{array}$ & $\begin{array}{l}\text { Guidance \& position } \\
\text { statement }\end{array}$ \\
\hline & Place of delivery & 1 & RCOG & Guidance \\
\hline & $\begin{array}{l}\text { Labor companion } \\
\text { (allow/limit at labor wards) }\end{array}$ & 2 & NHS, MFM Guidance & $\begin{array}{l}\text { Guidance \& Position } \\
\text { statement }\end{array}$ \\
\hline & Mode of delivery & 7 & $\begin{array}{l}\text { RCOG, FIGO, WHO, SMFM, } \\
\text { SOGC, ISUOG, ICM }\end{array}$ & $\begin{array}{l}\text { Guidance \& Position } \\
\text { statement, commentary }\end{array}$ \\
\hline & Intrapartum Fetal monitoring & 2 & SOGC, FIGO & Guidance \\
\hline & Shortening the second stage & 3 & FIGO, ICM, MFM & $\begin{array}{l}\text { Guidance, position } \\
\text { statement }\end{array}$ \\
\hline & $\begin{array}{l}\text { Immediate verses delayed cord } \\
\text { clamping }\end{array}$ & 5 & SMFM, FIGO, RCOG, ISUOG & $\begin{array}{l}\text { Guidance \& Position } \\
\text { statement, commentary }\end{array}$ \\
\hline & Mode of anesthesia for $C D$ & 2 & FIGO, SMFM & Guidance \\
\hline \multirow{4}{*}{ 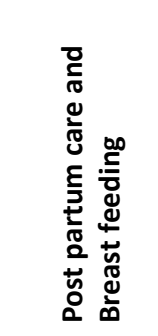 } & Skin-to-skin contact & 2 & FIGO, SOGC & Guidance \\
\hline & Maternal separation & 5 & $\begin{array}{l}\text { FIGO, SGOC, China } \\
\text { consensus, WHO, ISUOG }\end{array}$ & $\begin{array}{l}\text { Guidance, position } \\
\text { statement }\end{array}$ \\
\hline & Breast feeding & 3 & $\begin{array}{l}\text { ICM, WHO, China consensus } \\
\text { ISUOG }\end{array}$ & $\begin{array}{l}\text { Guidance, position } \\
\text { statement }\end{array}$ \\
\hline & $\begin{array}{l}\text { Post-natal visits } \\
\text { (virtual/ home based) }\end{array}$ & 4 & ACOG, RCOG, ICM, WHO & Guidance, Commentary \\
\hline
\end{tabular}


ment from the epi-center of the COVID-19 epidemic, China.

The findings of the documents were organized into recommendations in the domains of Antenatal care, labor and delivery, postnatal care, maternal isolation (Table 1). There were 9 recommendations in antenatal care; 8 in labor \& delivery and 4 regarding postnatal care and maternal isolation.

\section{The Key findings of the review are:}

Routine Antenatal care services should be delayed until a pregnant woman with COVID-19 recovers or tests negative twice.

$>$ Anatomic scanning is not a standard of practice but it may be considered in women who had the infection in the first trimester.

$>$ Growth monitoring is not universally recommended but it might be considered in those with ongoing pregnancy every 2-4 wks.

$>$ Antenatal corticosteroids can be administered in those women with preterm pregnancy ( $<34 \mathrm{wks}$ ) and mild illness. Its use in those with severe illness may be considered after consultation with infectious disease specialist.

$>$ Low dose Aspirin can be continued in women with COVID-19 infection if clinically indicated (e.g prevention of preeclampsia).

> Tocolytics, particularly NSAIDs should be used cautiously. Nifedipine is the preferred tocolytic agent.

$>$ Timing of delivery is dictated by maternal and fetal condition. Termination irrespective of gestational age is recommended in those with organ failure and in pts with severe respiratory failure.

$>$ Cesarean delivery is recommended in those with severe respiratory distress on ventilators, organ failure.

$>$ Shortening of the second stage is considered in COVID-19 parturient who are on face masks who will have difficulty of pushing.

$>$ A single, asymptomatic or screen negative birth partner be permitted to stay with the woman.

$>$ There is no consensus on immediate cord clamping.

$>$ Universal isolation of mother from the neonate is not a standard of practice. But it is reasonable to temporarily isolate severely ill woman and feed baby with expressed breast milk.

$>$ Postpartum care can be provided though telemedicine.

\section{Discussion}

\section{Antenatal care}

Antenatal care services are one of the essential services that any pregnant women should get irrespective of COVID-19 infection status. But there are limited guidelines on the provision of ANC to women with COVID-19. Both the
WHO and SOGC recommend considering to delay routine visits in pregnant women who are positive until they recover and in those who are being tested for COVID-19 until they test negative $[8,9]$. The FIGO recommends follow up appointments be postponed until 2 wks or until 2 negative results [10]. The mode of ANC services delivery should be modified, and innovative ways of care provision (such as telemedicine) are recommended with due consideration of individualized care plan. In those pregnant women with mild illness home confinement can be considered provided that this is possible logistically and that monitoring of the woman's condition can be ensured without compromising the safety of her family $[9,10]$.

\section{Anatomic scanning}

It is not still well known if COVID-19 infection is associated with congenital anomalies because of limited number of pregnant patients with COVID-19. The consensus statement from China obstetricians' society who have managed several pregnant mothers is to do anatomic scanning in those who have acquired the COVID-19 infection in the first trimester [11]. But the FIGO consensus guideline (interim guidance) recommends anatomic scanning at gestational age of 18 to 23 weeks for all women with COVID-19 infection [10].

\section{Antenatal surveillance}

A routine antenatal surveillance as a standard of practice is not as such recommended. Four of the guidelines have addressed the issue. The consensus statement from Chinese experts states that continuous EFM in the setting of severe illness should be considered only when delivery would not compromise maternal health [11]. Cardiotocography (CTG) for fetal heart rate (FHR) monitoring and ultrasound beyond gestational age of viability are recommended in those who have recovered from severe illness [8-10,12,13]. But the frequency of antenatal surveillance is not well stated.

\section{Growth monitoring}

One of the complications of COVID-19 infection in pregnancy is intrauterine growth restriction as a result of the viral pathogenic effects in the placental vasculature. Six of the guidelines have stated on importance of growth monitoring in ongoing pregnancies but they lack consistency on interval growth monitoring (frequency) $[9,11-15]$. ISUOG and ICM state monitoring every 4 wks while the SOGC and FIGO recommend every 2-4 wks. Both US for BPP and CTG/Doppler are options for fetal surveillance.

\section{Medication during pregnancy}

Antenatal corticosteroids: Generally, it is advised to use antenatal corticosteroids cautiously because of the potential effect of steroid in delaying viral clearance. Only 4 of the guidelines have addressed the issue and the use of corticosteroid in severely ill patients should be considered only after consultation with ID specialist $[10,13]$. But in those women with preterm pregnancy ( $<34$ wks.) and mild illness administration of a single course of antenatal steroids is recommended $[9,15]$. 
Low dose aspirin: The use of NSAID (esp. Ibuprofen) has been associated with a theoretical risk of facilitating COVID-19 infection. Only the MFM guidance published on journal of Obstetrics \& Gynecology has addressed the issue and recommended that it can be used in pregnant women for the prevention of preeclampsia [15].

Tocolytics: Clinical use of tocolytic agents was addressed in 2 of the guidelines. Tocolysis should not be used in an attempt to delay delivery in order to administer antenatal steroids [13]. The SMFM recommends that if tocolytics are indicated, Indomethacin should be avoided in face of uncertainty regarding NSAIDs and Nifedipine should be preferred over indomethacin [15].

The use of magnesium for seizure prophylaxis is also not recommended in severely ill patients with COVID-19 [15].

\section{Labor and delivery}

One of the challenges encountered in the management of pregnant women with COVID-19 is regarding labor and delivery care. This is because there are no strong clinical evidences to base on the standard of care for COVID-19 pregnant mothers.

\section{Timing and place of delivery}

Timing of delivery is generally recommended based on maternal \& fetal conditions. [10,11,15]. But pre-viable termination is recommended in those with organ failure and in pts with respiratory failure where improved oxygenation is expected with delivery of the fetus [11]. Timing of delivery in those with mild illness should not be dictated by maternal COVID-19 infection and in those pregnant mothers in 3rd trimester, it is better to wait until negative test results [11] or quarantine status is lifted in an attempt to avoid transmission to the neonate [15]. Place of delivery in COVID-19 infected mothers is addressed in only the RCOG guidance which states that hospital birth is preferred to home birth for women who have or are being tested for COVID-19, in light of the challenges associated with ensuring appropriate personal protective equipment in the home setting and the high rates of fetal distress that reported in the literature [12]. For the protection of the medical team, water birth should be avoided [13].

\section{Labor companionship}

One of the intriguing issues is labor companionship as there is a concern of transmission of covid to the companion and to the family. In institutional deliveries limiting the number of attendants is recommended but with due consideration of making sure that there is always a family member around in emergency situations. Six of the guidelines have recommended a single, asymptomatic or screen negative birth partner be permitted to stay with the woman [8,10-14].

\section{Conduct of labor and delivery}

Most of the guidelines recommend that Labor and delivery be conducted in isolated room if possible with negative pressure [13]. The room should be disinfected immediately after each delivery [10]. Guidance on intrapartum fetal moni- toring is very limited and has been addressed in only 2 of the guidelines $[9,10]$. Fetal monitoring in the form of EFM should be considered given evidence showing fetal distress during labor is common in women with COVID-19 infection [9]. Early epidural to minimize need for GA in the event of emergent CD is recommended in the MFM guidance [15]. There are no recommendations regarding rupture of membranes, oxytocin augmentation of labor and peculiarity in the definition of labor abnormalities. But, in the event that an infected woman has spontaneous onset of labor with optimal progress, she can be allowed to deliver vaginally [13].

\section{Shortening of the second stage}

The practice of instrumental delivery in the context of COVID-19 infection is addressed in only 2 of the documents revised. The China consensus statement and ISUOG state that shortening of the 2nd stage with instrumental delivery in those with respiratory distress who are on masks because active pushing while wearing a surgical mask may be difficult for the patient $[11,13]$. The ICM advocates against the practice of routine instrumental delivery [14]. With respect to a pregnant woman without a diagnosis of COVID-19 infection, but who might be a silent carrier of the virus, the ISUOG urges caution regarding the practice of active pushing while wearing a surgical mask, as it is unclear if there is an increased risk of exposure to any healthcare professional attending the delivery without PPE, because forceful exhalation may significantly reduce the effectiveness of a mask in preventing the spread of the virus by respiratory droplets [13].

\section{Cesarean delivery}

Regarding cesarean delivery most of the guidelines consistently state that it is reserved for routine obstetric indications $[9-12,14,15]$. But the ICM guidance emphasizes that patients' decision be respected and the China consensus statement advocates $C D$ in those mothers with severe respiratory distress in whom oxygenation might be improved by immediate delivery of the fetus [11]. Regional anesthesia as the choice of anesthesia for CD in COVID-19 positive mothers is recommended in the FIGO interim guidance [10] as it decreases staff exposure to COVID-19 but the ISUOG states both regional and general can be considered after consultation with anesthesiologists [13].

\section{Cord clamping}

There is no consensus on the practice of cord clamping. Four of the guidelines including RCOG \& ISUOG recommend avoiding delayed cord clamping although there is lack of clear evidence against it $[10,12,13]$. But guidance from the WHO and SOGC recommend to continue with the usual practice of delayed cord clamping as the theoretical risk of increased neonatal exposure to the Corona virus in the extra few minutes is negligible $[8,9]$.

Miscarried embryos/fetuses and placentae of COVID-19infected pregnant women should be treated as infectious tissues and they should be disposed of appropriately; if possible, testing of these tissues for COVID-19 by qRT-PCR should be undertaken [13]. 


\section{Breast feeding and maternal fetal bonding}

The practice of Skin-to-skin contact can be continued with the mother wearing a mask and after having washed her hands as stated in the FIGO and SOGC guidelines $[9,10]$. One of the controversial areas with no consistent recommendations is the practice breast feeding. The WHO and ICM have strongly recommended exclusive breast feeding in women with COVID-19 patients [8,14]. But the guidance didn't consider the severity of the illness. Rooming-in with Expressed breast milk (during time of separation) or suckling in mildly affected is recommended by ISUOG [13]. The necessary precautions should be made when woman with suspected/confirmed COVID-19 infection wants to breast feed: The mother should wear a face mask, avoid coughing or sneezing on the newborn. Standard COVID-19 hand washing principles should be followed prior to each feeding and prior to each time the mother touches the baby $[16,17]$.

Regarding maternal separation, the guidance from the WHO and RCOG do not generally recommend separation $[8,12]$. In the ISUOG interim guideline, If the mother is severely or critically ill, separation appears to be the best option, with attempts to express breastmilk in order to maintain milk production. Precautions should be taken when cleaning the breast pumps and the option of using a screen negative relative wearing appropriate protective equipments to pump breast are recommended [13]. The Canadian society of Obstetrics and Gynecology guidance does not recommend universal isolation of the infant from either confirmed of suspected infection in the mother. However, depending on a family's values and availability of resources they may choose to separate infant from mother until isolation precautions for the mother can be formally discontinued [9].

\section{Post-natal care}

Modification of postnatal services is recommended with decreased number of visits and provision of care with telehealth. The majority of postpartum visits may be conducted remotely as long as the patient does not have specific concerns that require in-person examination [10].

Limitations of the review: Guidelines and practice recommendations from developing could not be retrieved \& included in the review. Most of the documents that are included in this review didn't pass through rigorous guideline development process because of the nature of the pandemics.

\section{Conclusions}

Obstetric care provision for COVID-19 infected mothers is primarily based on limited experience from case reports and expert opinions. Updated guidance for clinical practice are imperative as new scientific evidence emerges.

\section{Declarations}

\section{Ethics approval and consent to participate}

Not applicable.

\section{Consent for publication}

Not applicable.

\section{Availability of data and materials}

All materials and data are included in the manuscript (additional files).

\section{Competing interests}

The authors declare that they have no any competing interests.

\section{Funding}

There were no any funding sources for all activities related with the preparation of the review.

\section{Authors' contributions}

Both WGJ and LTB were directly involved in the inception, collection of relevant resources, data extraction. WGJ did the write up of the final manuscript; LBT revised it. All authors have read and approved the manuscript.

\section{Acknowledgements}

None.

\section{References}

1. Adhikari SP, Meng S, Wu YJ, et al. (2020) Epidemiology, causes, clinical manifestation and diagnosis, prevention and control of coronavirus disease (COVID-19) during the early outbreak period: A scoping review. Infect Dis Poverty 9: 29.

2. World Health Organization (2020) Statement on the second meeting of the International Health Regulations (2005) Emergency Committee regarding the outbreak of novel coronavirus (2019-nCoV).

3. World Health Organization (2020) WHO Director-General's opening remarks at the media briefing on COVID-19 - 13 March 2020.

4. Zhu N, Zhang D, Wang W, et al. (2020) A novel coronavirus from patients with pneumonia in China, 2019. N Engl J Med 382: 727733.

5. Zhang L, Jiang $Y$, Wei M, et al. (2020) Analysis of the pregnancy outcomes in pregnant women with COVID-19 in Hubei Province. Zhonghua Fu Chan Ke Za Zhi 55: 166-171.

6. Wen R, Sun $Y$, Xing QS (2020) A patient with SARS-CoV-2 infection during pregnancy in Qingdao, China. J Microbiol Immunol Infect 53: 499-500.

7. Li N, Han L, Peng M, et al. (2020) Maternal and neonatal outcomes of pregnant women with Coronavirus Disease 2019 (COVID-19) pneumonia: A case-control study. Clin Infect Dis 71: 2035-2041.

8. World Health Organization (2020) Clinical management of severe acute respiratory infection (SARI) when COVID-19 disease is suspected. Interim guidance.

9. Elwood C, Raeside A, Boucoiran I (2020) Updated SOGC Committee Opinion - COVID-19 in Pregnancy. J Obstet Gynaecol Can.

10. Poon LC, Yang H, Kapur A, et al. (2020) Global interim guidance on coronavirus disease 2019 (COVID-19) during pregnancy and puerperium from FIGO and allied partners: Information for healthcare professionals. Int J Gynaecol Obstet 149: 273-286.

11. Liang H, Acharya G (2020) Novel corona virus disease (COVID-19) 
in pregnancy: What clinical recommendations to follow? Acta Obstet Gynecol Scand 99: 439-442.

12. Ortiz EI, Herrera E, Torre A (2020) Coronavirus (COVID-19) infection in pregnancy. Colomb Med (Cali) 51: e4271.

13. ISUOG (2020) Guidance on COVID-19 during pregnancy and the puerperium. International Society of Ultrasound in Obstetrics and Gynecology.
14. ICM (2020) Women's rights in childbirth must be upheld during the coronavirus pandemic. International Confederation of Midwives.

15. Boelig RC, Manuck T, Oliver EA, et al. (2020) Labor and delivery guidance for COVID-19. Am J Obstet Gynecol MFM 2: 100110.

16. https://www.cdc.gov/coronavirus/2019-ncov/need-extra-precautions/pregnancy-breastfeeding.html

17. COVID-19 FAQs for Obstetrician-Gynecologists, Obstetrics. 


\section{Appendix}

\section{Appendix 1:}

- Title of data: Search strategy for retrieving the relevant documents.

- Description of data: This is a table showing the key words used in browsing data bases and the number of records retrieved.

\section{Appendix 2:}

- Title of Data: Data Extraction format.
- Description of data: This is a simple data extraction format organized based on the areas of obstetric care provision to pregnant women.

\section{Appendix 3:}

Title of data: Preferred Reporting Items for Systematic reviews and Meta-Analyses extension for Scoping Reviews (PRISMA-ScR) Checklist.

Description of data: This is the filled form of PRISMA-ScR checklist for the review on Obstetric Care provision of Women with COVID-19 infection. 